\title{
Synthesis and pharmacological evaluation of pyrazolopyrimidopyrimidine derivatives: anti-inflammatory agents with gastroprotective effect in rats
}

\author{
Amine Karoui · Fatma Allouche - Monia Deghrigue - Asma Agrebi • \\ Abderrahman Bouraoui · Fakher Chabchoub
}

Received: 17 April 2013/Accepted: 17 August 2013/Published online: 4 September 2013

(C) The Author(s) 2013. This article is published with open access at Springerlink.com

\begin{abstract}
We report the synthesis of new anti-inflammatory 1,7-dihydropyrazolo $\left[3^{\prime}, 4^{\prime}: 4,5\right]$ pyrimido $[1,6-a]$ pyrimidine 5 from aminocyanopyrazole. All compounds were characterized by physical, chemical and spectral studies. Preliminary pharmacological evaluation of the resulting products showed that compounds $5 \mathbf{a}, \mathbf{b}, \mathbf{f}(50-100 \mathrm{mg} / \mathrm{kg}$, i.p) are active anti-inflammatory agents in carrageenaninduced rat paw oedema assay, and their effects are comparable to that of acetylsalicylic-lysine (300 mg/kg, i.p.), used as a reference drug. The nature of substituent $\left(Y, R_{3}\right)$ had a pronounced effect on the anti-inflammatory activity. Studies of structure-activity relationships have led to selection of compound ethyl-3,5-dimethyl-7-imino- $N^{1}$-phenyl-1,7-dihydropyrazolo $\left[3^{\prime}, 4^{\prime}: 4,5\right]$ pyrimido[1,6- $\left.a\right]$ pyrimidine-6-carboxylate, 5f which exhibited the most potent antiinflammatory activity. In addition, the compounds $\mathbf{5 a}, \mathbf{b}$, f showed a significant gastroprotective effect against $\mathrm{HCl}$ / EtOH-induced gastric ulcer.
\end{abstract}

Keywords Aminocyanopyrazole · Anti-inflammatory · Gastroprotective · Pyrazolo $[3,4-d]$ pyrimidine . Dihydropyrazolo $\left[3^{\prime}, 4^{\prime}: 4,5\right]$ pyrimido $[1,6-a]$ pyrimidine

A. Karoui · F. Allouche $(\bowtie) \cdot A$. Agrebi · F. Chabchoub Laboratoire de Chimie Appliquée, Hétérocycles, Corps Gras et Polymères, Faculté des Sciences de Sfax, Université de Sfax, 3018 Sfax, Tunisia

e-mail: fatmaallouch@yahoo.fr

M. Deghrigue $\cdot$ A. Bouraoui

Unité de Recherche des Substances Actives Marines (URSAM), Laboratoire de Pharmacologie, Faculté de Pharmacie, Université de Monastir, 5000 Monastir, Tunisia

\section{Introduction}

Nonsteroidal anti-inflammatory drugs (NSAIDs) are most widely used to treat variety of acute and chronic inflammatory diseases. Such drugs are being increasingly used for the treatment of postoperative pain (Moote, 1992) with or without supplemental opioid agents. The pharmacological action of these agents was assigned to inhibit two enzymes, known as cyclooxygenase-1 (COX-1) and cyclooxygenase2 (COX-2) (Vane et al., 1998). The constitutive isoform COX-1 is present in most tissues and is involved in the synthesis of prostaglandins vital to normal cell function. In contrast, the inducible isoform COX-2 appears to be produced primarily in response to growth factors or inflammatory mediators, such as cytokines (Vane and Botting, 1996). Many of the currently available NSAIDs, including indomethacin and piroxicam, are more potent inhibitors of COX-1 than that of COX-2 (Vane and Botting, 1995). This preferential inhibition of COX-1 may be responsible for many of the adverse effects associated with NSAIDs. It has been postulated that NSAIDs which preferentially inhibit COX-2, such as meloxicam (Lipscomb et al., 1998), celecoxib (Simon et al., 1998) and several experimental drugs including NS 398, L-745,337 and DFP, should produce the same or better anti inflammatory effects with less gastrointestinal, haematological and renal toxicities than classical NSAIDs (Winter et al., 1962). Pyrazolopyrimidines are a class of sedative and anxiolytic drugs such as Zaleplon known by its hypnotic effect (Weitzel et al., 2000). However, pyrazolopyrimidine derivatives become a new chemical resource for searching of novel bioactive compounds in drug development.

On this basis, we directed our attention to the synthesis of novel 1,7-dihydropyrazolo $\left[3^{\prime}, 4^{\prime}: 4,5\right]$ pyrimido[1,6- $\left.a\right]$ pyrimidines $5 \mathbf{a}-\mathbf{i}$ related to aminocyanopyrazole with the 
aim of improving their anti-inflammatory activity and reducing their ulcerogenic properties as it appeared to be plausible that variation of the active compound structures could exert a pronounced influence on activity, as the case with $\mathbf{5 b}, \mathbf{f}$.

\section{Materials and methods}

\section{Chemistry}

Phenyl hydrazine, malononitrile, triethylorthoester and ammoniac were purchased from Sigma Chemical (Berlin, Germany). Analytical grade solvents (ethanol, $\mathrm{HCl}$, ethyl acetate, chloroform) were obtained from Merck.

Melting points (mp) were determined on a Buchi capillary apparatus and were uncorrected. Nuclear magnetic resonance (NMR) spectra were recorded on a Bruker 300 spectrometer $\left({ }^{1} \mathrm{H}\right.$ at $300 \mathrm{MHz}$ and ${ }^{13} \mathrm{C}$ at $\left.75 \mathrm{MHz}\right)$ with deuterio-dimethylsulphoxide ( $d$-DMSO) as solvent and tetramethylsilane as internal standard reference. Infra-red (IR) spectra were recorded on a Bio-rad FTS-6000 spectrometer. Solvents used in reactions were dried and distilled before use. The purity of all synthesized compounds was controlled by thin layer chromatography (TLC; Merck silica gel plates $60 \mathrm{~F}-254)$. High resolution masses were recorded on a spectrometer JEOL JMS-Gcmate II is composed of a GC/MS system from compounds dissolved in dichloromethane.

\section{Synthesis and spectral data of compounds 2-5}

\section{5-Amino-4-cyano- $N^{1}$-phenyl pyrazoles (2) 5-Amino-4-} cyano-1- $N^{1}$-phenyl pyrazoles prepared via a standard addition of hydrazine derivatives to ketene ethoxymethylene compounds following the reported procedure. Recrystallization from ethanol afforded pure $\mathbf{2}$ in good yields.

4-Cyano- $N^{I}$-phenyl pyrazolo-5-imidates (3) The required 5-amino-4-cyano- $N^{I}$-phenyl pyrazole $(1.0 \mathrm{mmol})$ was treated with triethylorthoester $6.0 \mathrm{mmol}$ ) and a catalytic amount of acetic acid and the mixture was refluxed for $24 \mathrm{~h}$. After cooling, the reaction mixture was evaporated. The product was filtered, washed with diethyl ether then purified by recrystallisation (ethanol) (Gupta et al., 2008; Allouche et al., 2013).

\section{4-Amino- $N^{I}$-phenyl pyrazolo[3,4-d]pyrimidine (4) A} solution of imidate $3(1.0 \mathrm{mmol})$ in dry ethanol $(5 \mathrm{ml})$ was treated with ammoniac $(2.0 \mathrm{mmol})$ and a catalytic amount of acetic acid. The reaction mixture was refluxed for $6 \mathrm{~h}$, and the formed solid was collected by filtration, dried and recrystallized from ethanol to give compound 4 . a) 4-Amino- $N^{I}$-phenyl-1H-pyrazolo[3,4-d]pyrimidine $4 a$ Yield $83 \%$; mp $228{ }^{\circ} \mathrm{C}$; IR $\left(\mathrm{cm}^{-1}\right) ; v_{\mathrm{NH} 2} 3100,3283$; $v_{\mathrm{C}=\mathrm{N}} 1480,1500,1590 \mathrm{~cm}^{-1}$; $\mathrm{RMN}{ }^{1} \mathrm{H}(\delta \mathrm{ppm}$, DMSO): $4.69\left(2 \mathrm{H}, \mathrm{s}, \mathrm{NH}_{2}\right), 7.36(1 \mathrm{H}, \mathrm{t}, J=7.3 \mathrm{~Hz}$, $\left.\mathrm{ArH}_{4}\right), 7.48\left(2 \mathrm{H}, \mathrm{t}, J=7.3 \mathrm{~Hz}, \mathrm{ArH}_{3}\right.$ and $\left.\mathrm{ArH}_{5}\right), 7.52$ $\left(2 \mathrm{H}, \mathrm{d}, J=7.3 \mathrm{~Hz}, \mathrm{ArH}_{2}\right.$ and $\left.\mathrm{ArH}_{6}\right), 7.60\left(1 \mathrm{H}, \mathrm{s}, \mathrm{H}_{3}\right)$, $7.72\left(1 \mathrm{H}, \mathrm{s}, \mathrm{H}_{6}\right),{ }^{13} \mathrm{C}$ RMN ( $\delta$ ppm, DMSO): 114.14 (C-3a), $124.27\left(\mathrm{C}-2^{\prime}\right.$ and $\left.\mathrm{C}-6^{\prime}\right), 129.00\left(\mathrm{C}-4^{\prime}\right), 129.58$ (C-3' and C-5'), 130.04 (C-3), $136.94\left(\mathrm{C}-1^{\prime}\right), 141.36$ (C-7a), 149.83 (C-6), 156.84 (C-4); HRMS Calcd. for $\mathrm{C}_{11} \mathrm{H}_{9} \mathrm{~N}_{5}: 211.0858$, found: 211.0859 .

b) 4-Amino-3-methyl- $N^{1}$-phenyl-1H-pyrazolo [3,4-d] pyrimidine $4 \boldsymbol{b}$ Yield $68 \%$; $\mathrm{mp} 192{ }^{\circ} \mathrm{C}$; IR $\left(\mathrm{cm}^{-1}\right)$; $v_{\mathrm{NH} 2} 3083,3317 ; v_{\mathrm{C}=\mathrm{N}} 1626,1647,1665 ; \mathrm{RMN}^{1} \mathrm{H}$ $(\delta \mathrm{ppm}, \mathrm{DMSO}): 2.76\left(3 \mathrm{H}, \mathrm{s}, \mathrm{CH}_{3}\right), 5.97(2 \mathrm{H}, \mathrm{s}$, $\left.\mathrm{NH}_{2}\right), 7.33\left(1 \mathrm{H}, \mathrm{t}, J=7.1 \mathrm{~Hz}, \mathrm{ArH}_{4}\right), 7.57(2 \mathrm{H}, \mathrm{t}$, $J=7.1 \mathrm{~Hz}, \mathrm{ArH}_{3}$ and $\left.\mathrm{ArH}_{5}\right), 8.16(2 \mathrm{H}, \mathrm{d}$, $J=7.1 \mathrm{~Hz}, \mathrm{ArH}_{2}$ and $\left.\mathrm{ArH}_{6}\right), 8.46\left(1 \mathrm{H}, \mathrm{s}, \mathrm{H}_{3}\right)$; $\mathrm{RMN}^{13} \mathrm{C}(\delta \mathrm{ppm}, \mathrm{DMSO}): 14.89\left(\mathrm{CH}_{3}\right), 101.23(\mathrm{C}-$ 3a), 121.49 (C-2' and C-6' $), 126.37\left(\mathrm{C}-4^{\prime}\right), 129.19$ $\left(\mathrm{C}-3^{\prime}\right.$ and $\left.\mathrm{C}-5^{\prime}\right), 138.81$ (C-3), $141.83\left(\mathrm{C}-1^{\prime}\right)$, 154.41 (C-7a), 156.48 (C-4), 158.40 (C-6); HRMS Calcd. for $\mathrm{C}_{12} \mathrm{H}_{11} \mathrm{~N}_{5}: 225.1014$, found: 225.1018 .

c) 4-Amino-6-methyl- $N^{1}$-phenyl-1H-pyrazolo $[3,4-d]$ pyrimidine $4 c$ Yield $70 \%$; mp $160{ }^{\circ} \mathrm{C}$; IR $\left(\mathrm{cm}^{-1}\right)$; $v_{\mathrm{NH} 2} 3090,3320 ; v_{\mathrm{C}=\mathrm{N}} 1597,1638,1663 ; \mathrm{RMN}^{1} \mathrm{H}$ $(\delta \mathrm{ppm}, \mathrm{DMSO}): 2.65\left(3 \mathrm{H}, \mathrm{s}, \mathrm{CH}_{3}\right), 4.28(2 \mathrm{H}, \mathrm{s}$, $\left.\mathrm{NH}_{2}\right), 7.28\left(1 \mathrm{H}, \mathrm{t}, J=7.3 \mathrm{~Hz}, \mathrm{ArH}_{4}\right), 7.56(2 \mathrm{H}, \mathrm{t}$, $J=7.3 \mathrm{~Hz}, \quad \mathrm{ArH}_{3}$ and $\left.\mathrm{ArH}_{5}\right), 8.19(2 \mathrm{H}, \mathrm{d}$, $J=7.3 \mathrm{~Hz}, \mathrm{ArH}_{2}$ and $\left.\mathrm{ArH}_{6}\right), 8.29\left(1 \mathrm{H}, \mathrm{s}, \mathrm{H}_{6}\right)$; $\mathrm{RMN}^{13} \mathrm{C}(\delta \mathrm{ppm}, \mathrm{DMSO}): 14.44\left(\mathrm{CH}_{3}\right), 100.24(\mathrm{C}-$ $3 \mathrm{a}), \mathrm{C}_{\text {arom }} 120.24\left(\mathrm{C}-2^{\prime}\right.$ and $\left.\mathrm{C}-6^{\prime}\right), 124.67\left(\mathrm{C}-4^{\prime}\right)$, $129.16\left(\mathrm{C}-3^{\prime}\right.$ and $\left.\mathrm{C}-5^{\prime}\right), 138.8(\mathrm{C}-3), 142.79\left(\mathrm{C}-1^{\prime}\right)$; $\mathrm{C}_{3} 154.14 \quad$ (C-7a), $156.51 \quad(\mathrm{C}-4), 158.58 \quad$ (C-6); HRMS Calcd. for $\mathrm{C}_{12} \mathrm{H}_{11} \mathrm{~N}_{5}: 225.1014$, found: 225.1016.

7-Imino- $N^{1}$-phenyl-1,7-dihydropyrazolo [3', 4':4,5]pyrimido [1,6-a]pyrimidine 5a-e A mixture of compound 4 (1.0 mmol), ketene ethoxymethylene compounds 1 or ethyl-2cyano-3-ethoxyalkyl-2-enoate $(1.0 \mathrm{mmol})$ and a catalytic amount of acetic acid was refluxed for $2 \mathrm{~h}$ in $10 \mathrm{ml}$ ethanol. The formed precipitate was filtered, washed by diethyl ether, dried and recrystallized from ethanol to give compound 5 in good yield.

a) 6-Cyano-7-imino-3-methyl- $N^{1}$-phenyl-1,7-dihydropyrazolo[ $\left.3^{\prime}, 4^{\prime}: 4,5\right]$ pyrimido[1,6-a]pyrimidine $5 \boldsymbol{a}$ Yield $68 \%$; mp $290{ }^{\circ} \mathrm{C}$; IR $\left(\mathrm{cm}^{-1}\right) ; v_{\mathrm{NH}} 3356 ; v_{\mathrm{C} \equiv \mathrm{N}} 2212$; $v_{\mathrm{C}=\mathrm{N}} 1534,1554,1587$; RMN ${ }^{1} \mathrm{H}(\delta \mathrm{ppm}, \mathrm{DMSO})$ : $2.51\left(3 \mathrm{H}, \mathrm{s}, \mathrm{CH}_{3}\right) ; 7.38\left(1 \mathrm{H}, \mathrm{t}, J=7.3 \mathrm{~Hz}, \mathrm{ArH}_{4}\right)$; $7.53\left(2 \mathrm{H}, \mathrm{t}, J=7.3 \mathrm{~Hz}, \mathrm{ArH}_{3}\right.$ and $\left.\mathrm{ArH}_{5}\right) ; 7.71(2 \mathrm{H}$, $\mathrm{d}, J=7.3 \mathrm{~Hz}, \mathrm{ArH}_{2}$ and $\left.\mathrm{ArH}_{6}\right) ; 8.02\left(1 \mathrm{H}, \mathrm{s}, \mathrm{H}_{5}\right)$; 
$8.38\left(1 \mathrm{H}, \mathrm{s}, \mathrm{H}_{9}\right) ; 8.66(1 \mathrm{H}, \mathrm{s}, \mathrm{NH}) ; \mathrm{RMN}^{13} \mathrm{C}(\delta \mathrm{ppm}$, DMSO): $14.64\left(\mathrm{CH}_{3}\right) ; 91.81$ (C-6); 105.88 (C-3a); $116.24(\mathrm{CN}) ; \mathrm{C}_{\text {arom }} 120.46\left(\mathrm{C}-2^{\prime}\right.$ and $\left.\mathrm{C}-6^{\prime}\right), 124.17$ (C-4'), $129.27\left(\mathrm{C}-3^{\prime}\right.$ and $\left.\mathrm{C}-5^{\prime}\right), 137.89\left(\mathrm{C}-1^{\prime}\right), 143.42$ (C-10a), 149.71 (C-3),159.61 (C-5),161.88 (C-9), 162.15 (C-4a); 163.43 (C-7); HRMS Calcd. for $\mathrm{C}_{16} \mathrm{H}_{11} \mathrm{~N}_{7}$ :301.1076, found: 301.1051 .

b) 6-Cyano-7-imino-3,5-dimethyl- $N^{1}$-phenyl-1, 7-dihydropyrazolo $\left[3^{\prime}, 4^{\prime}: 4\right.$, 5]pyrimido[1, 6-a]pyrimidine $\mathbf{5 b}$ Yield $54 \%$; mp $182{ }^{\circ} \mathrm{C}$; IR $\left(\mathrm{cm}^{-1}\right): v_{\mathrm{NH}} 3324 ; v_{\mathrm{C} \equiv \mathrm{N}}$ 2230; $v_{\mathrm{C}=\mathrm{N}} 1509,1562,1586 ; \mathrm{RMN}^{1} \mathrm{H}(\delta \mathrm{ppm}$, DMSO): $2.50\left(3 \mathrm{H}, \mathrm{s}, \mathrm{CH}_{3}\right), 2.64\left(3 \mathrm{H}, \mathrm{s}, \mathrm{CH}_{3}\right) ; 7.26$ $\left(1 \mathrm{H}, \mathrm{t}, J=7.3 \mathrm{~Hz}, \mathrm{ArH}_{4}\right) ; 7.51(2 \mathrm{H}, \mathrm{t}, J=7.3 \mathrm{~Hz}$, $\mathrm{ArH}_{3}$ and $\left.\mathrm{ArH}_{5}\right) ; 7.54\left(2 \mathrm{H}, \mathrm{d}, J=7.3 \mathrm{~Hz}, \mathrm{ArH}_{2}\right.$ and $\left.\mathrm{ArH}_{6}\right) ; 8.19\left(1 \mathrm{H}, \mathrm{s}, \mathrm{H}_{9}\right) ; 8.27(1 \mathrm{H}, \mathrm{s}, \mathrm{NH}) ; \mathrm{RMN}^{13} \mathrm{C}(\delta$ ppm, DMSO): $14.42\left(\mathrm{CH}_{3}\right) ; 21.00\left(\mathrm{CH}_{3}\right) ; 87.23(\mathrm{C}-$ 6); 100.25 (C-3a); $109.00(\mathrm{CN}) ; 120.22\left(\mathrm{C}-2^{\prime}\right.$ and C-6' $\left.{ }^{\prime}\right), 125.51\left(\mathrm{C}-4^{\prime}\right), 128.98\left(\mathrm{C}-3^{\prime}\right.$ and $\left.\mathrm{C}-5^{\prime}\right), 138.89$ $\left(\mathrm{C}-1^{\prime}\right)$; 142.79 (C-10a); 154.17 (C-3), 156.49 (C-5), 164.59 (C-9), 165.71 (C-4a), 167.94 (C-7); HRMS Calcd. for $\mathrm{C}_{17} \mathrm{H}_{13} \mathrm{~N}_{7}: 315.1232$, found: 315.1214 .

c) 6-Cyano-7-imino-9-methyl- $N^{1}$-phenyl-1,7-dihydropyrazolo $\left[3^{\prime}, 4^{\prime}: 4,5\right]$ pyrimido[1,6-a]pyrimidine $5 \boldsymbol{c}$ Yield $71 \%$; mp $166{ }^{\circ} \mathrm{C}$; IR $\left(\mathrm{cm}^{-1}\right) ; v_{\mathrm{NH}} 3321.86 ; v_{\mathrm{C} \equiv \mathrm{N}}$ 2223, 1536, 1561, 1599; RMN ${ }^{1} \mathrm{H}(\delta$ ppm, DMSO): $2.62\left(3 \mathrm{H}, \mathrm{s}, \mathrm{CH}_{3}\right) ; 7.40\left(1 \mathrm{H}, \mathrm{t}, J=7.3 \mathrm{~Hz}, \mathrm{ArH}_{4}\right)$; $7.49\left(2 \mathrm{H}, \mathrm{t}, J=7.3 \mathrm{~Hz}, \mathrm{ArH}_{3}\right.$ and $\left.\mathrm{ArH}_{5}\right) ; 7.68(2 \mathrm{H}, \mathrm{d}$, $J=7.3 \mathrm{~Hz}, \mathrm{ArH}_{2}$ and $\left.\mathrm{ArH}_{6}\right) ; 8.19\left(1 \mathrm{H}, \mathrm{s}, \mathrm{H}_{5}\right) ; 8.41$ $\left(1 \mathrm{H}, \mathrm{s}, \mathrm{H}_{9}\right) ; 8.73(1 \mathrm{H}, \mathrm{s}, \mathrm{NH}) ; \mathrm{RMN}^{13} \mathrm{C}(\delta \mathrm{ppm}$, DMSO): $14.32\left(\mathrm{CH}_{3}\right) ; 89.64$ (C-6); 103.64 (C-3a); $111.83(\mathrm{CN}) ; \mathrm{C}_{\text {arom }} 120.38\left(\mathrm{C}-2^{\prime}\right.$ and $\left.\mathrm{C}-6^{\prime}\right), 126.65(\mathrm{C}-$ $\left.4^{\prime}\right), 138.42\left(\mathrm{C}-3^{\prime}\right.$ and $\left.\mathrm{C}-5^{\prime}\right), 140.12\left(\mathrm{C}-1^{\prime}\right), 143.42(\mathrm{C}-$ 10a),141.69 (C-3),148.47 (C-5),160.28 (C-9), 161.92 (C-4a); 162.00 (C-7). $\mathrm{C}_{16} \mathrm{H}_{11} \mathrm{~N}_{7}, 301.1051$; HRMS Calcd. for $\mathrm{C}_{16} \mathrm{H}_{11} \mathrm{~N}_{7}$ : 301.1076, found: 301.1087 .

d) 6-Cyano-7-imino- $N^{1}$-phenyl-1,7-dihydropyrazolo[ $3^{\prime}, 4^{\prime}$ : 4,5]pyrimido[1,6-a]pyrimidine 5d Yield $77 \%$; mp $248{ }^{\circ} \mathrm{C}$; IR $\left(\mathrm{cm}^{-1}\right) ; v_{\mathrm{NH}} 3189 ; v_{\mathrm{C} \equiv \mathrm{N}} 2250 ; v_{\mathrm{C}=\mathrm{N}} 1532$, 1559, 1562; RMN ${ }^{1} \mathrm{H}(\delta$ ppm, DMSO): $7.33(1 \mathrm{H}, \mathrm{t}$, $\left.J=7.3 \mathrm{~Hz}, \mathrm{ArH}_{4}\right), 7.55\left(2 \mathrm{H}, \mathrm{t}, J=7.3 \mathrm{~Hz}, \mathrm{ArH}_{3}\right.$ and $\left.\mathrm{ArH}_{5}\right), 8.03\left(1 \mathrm{H}, \mathrm{s}, \mathrm{H}_{5}\right), 8.21\left(2 \mathrm{H}, \mathrm{d}, J=7.3 \mathrm{~Hz}, \mathrm{ArH}_{2}\right.$ and $\left.\mathrm{ArH}_{6}\right), 8.31\left(1 \mathrm{H}, \mathrm{s}, \mathrm{H}_{9}\right), 8.36\left(1 \mathrm{H}, \mathrm{s}, \mathrm{H}_{3}\right), 8.37(1 \mathrm{H}, \mathrm{s}$, $\mathrm{NH}) ; \mathrm{RMN}^{13} \mathrm{C}(\delta \mathrm{ppm}, \mathrm{DMSO}): 89.87(\mathrm{C}-6) ; 101.37(\mathrm{C}-$ 3a); $120.45(\mathrm{CN}) ; \mathrm{C}_{\text {arom }} 126.00\left(\mathrm{C}-2^{\prime}\right.$ and $\left.\mathrm{C}-6^{\prime}\right), 129.10$ $\left(\mathrm{C}-4^{\prime}\right), 13015\left(\mathrm{C}-3^{\prime}\right.$ and $\left.\mathrm{C}-5^{\prime}\right), 134.04\left(\mathrm{C}-1^{\prime}\right) ; 138.94(\mathrm{C}-$ 10a); 139.11 (C-3); 142.14 (C-5);153.19 (C-9); 156.68 (C-4a); 158.26 (C-7); HRMS Calcd. for $\mathrm{C}_{15} \mathrm{H}_{9} \mathrm{~N}_{7}$ : 287.0976, found: 287.0919 .

e) 6-Cyano-7-imino-5-ethyl- $N^{1}$-phenyl-1,7-dihydropyrazolo $\left[3^{\prime}, 4^{\prime}: 4,5\right]$ pyrimido[1,6-a]pyrimidine $5 \boldsymbol{e}$ Yield $70 \%$; mp $168{ }^{\circ} \mathrm{C}$; IR $\left(\mathrm{cm}^{-1}\right) ; v_{\mathrm{NH}} 3332 ; v_{\mathrm{C} \equiv \mathrm{N}} 2218$; $v_{\mathrm{C}=\mathrm{N}} 1568,1589,1620 ; \mathrm{RMN}^{1} \mathrm{H}(\delta \mathrm{ppm}, \mathrm{DMSO}): 1.23$ $\left(3 \mathrm{H}, \mathrm{t}, \mathrm{CH}_{3}\right) ; 2.30\left(2 \mathrm{H}, \mathrm{q}, \mathrm{CH}_{2}\right) ; 7.30(1 \mathrm{H}, \mathrm{t}, J=7.3 \mathrm{~Hz}$,
$\left.\mathrm{ArH}_{4}\right) ; 7.52\left(2 \mathrm{H}, \mathrm{t}, J=7.3 \mathrm{~Hz}, \mathrm{ArH}_{3}\right.$ and $\left.\mathrm{ArH}_{5}\right) ; 8.04$ $\left(2 \mathrm{H}, \mathrm{d}, J=7.3 \mathrm{~Hz}, \mathrm{ArH}_{2}\right.$ and $\left.\mathrm{ArH}_{6}\right) ; 8.18\left(1 \mathrm{H}, \mathrm{s}, \mathrm{H}_{5}\right)$; $8.52\left(1 \mathrm{H}, \mathrm{s}, \mathrm{H}_{9}\right) ; 11.16(1 \mathrm{H}, \mathrm{s}, \mathrm{NH}) ; \mathrm{RMN}^{13} \mathrm{C}(\delta \mathrm{ppm}$, DMSO): $9.01\left(\mathrm{CH}_{3}\right): 29.31\left(\mathrm{CH}_{2}\right) ; 92.54(\mathrm{C}-6) ; 106.31$ (C-3a); $114.07(\mathrm{CN}) ; \mathrm{C}_{\text {arom }} 121.28\left(\mathrm{C}-2^{\prime}\right.$ and $\left.\mathrm{C}^{\prime} 6^{\prime}\right)$, $124.73\left(\mathrm{C}-4^{\prime}\right), 126.56\left(\mathrm{C}-3^{\prime}\right.$ and $\left.\mathrm{C}-5^{\prime}\right), 141.13(\mathrm{C}-$ $\left.1^{\prime}\right), 145.82$ (C-10a), 152.63 (C-3),155.28 (C-9), 161.23 (C-4a), 162.07 (C-7); 165.49 (C-5); HRMS Calcd. for $\mathrm{C}_{17} \mathrm{H}_{13} \mathrm{~N}_{7}: 315.1232$, found: 315.1352 .

f) Ethyl-3,5-dimethyl-7-imino- $N^{1}$-phenyl-1,7-dihydropyrazolo [3', 4':4,5]pyrimido[1,6-a]pyrimidine-6-carboxylate $5 f$ Yield $71 \%$; mp $170{ }^{\circ} \mathrm{C}$; IR $\left(\mathrm{cm}^{-1}\right) ; v_{\mathrm{NH}}$ $3081 ; v_{\mathrm{CO}} 1747 ; v_{\mathrm{C}=\mathrm{N}} 1510,1565,1590 ; \mathrm{RMN}{ }^{1} \mathrm{H}(\delta$ ppm, DMSO) $1.21\left(3 \mathrm{H}, \mathrm{t}, J=7.2 \mathrm{~Hz}, \mathrm{CH}_{3}\right) ; 1.91$ $\left(3 \mathrm{H}, \mathrm{s}, \mathrm{CH}_{3}\right) ; 2.62\left(3 \mathrm{H}, \mathrm{s}, \mathrm{CH}_{3}\right) ; 4.15(2 \mathrm{H}, \mathrm{q}$, $\left.J=7.2 \mathrm{~Hz}, \mathrm{CH}_{2}\right) ; 7.28\left(1 \mathrm{H}, \mathrm{t}, J=7.3 \mathrm{~Hz}, \mathrm{ArH}_{4}\right)$; $7.51\left(2 \mathrm{H}, \mathrm{t}, J=7.3 \mathrm{~Hz}, \mathrm{ArH}_{3}\right.$ and $\left.\mathrm{ArH}_{5}\right) ; 8.17(2 \mathrm{H}, \mathrm{d}$, $J=7.3 \mathrm{~Hz}, \mathrm{ArH}_{2}$ and $\left.\mathrm{ArH}_{6}\right) ; 8.26\left(1 \mathrm{H}, \mathrm{s}, \mathrm{H}_{9}\right) ; 11.97$ $(1 \mathrm{H}, \mathrm{s}, \mathrm{NH}) . \mathrm{RMN}^{13} \mathrm{C}(\delta \mathrm{ppm}, \mathrm{DMSO}) 13.01\left(\mathrm{CH}_{3}\right)$; $14.00\left(\mathrm{CH}_{3}\right) ; 24.45\left(\mathrm{CH}_{3}\right) ; 66.03\left(\mathrm{CH}_{2}\right) ; 105.28(\mathrm{C}-$ 6); 115.10 (C-3a); 121.07 (C-2' and C-6'), 125.50 (C$\left.4^{\prime}\right), 129.12\left(\mathrm{C}-3^{\prime}\right.$ and $\left.\mathrm{C}-5^{\prime}\right), 138.88\left(\mathrm{C}-1^{\prime}\right), 142.79(\mathrm{C}-$ 10a), 146.88 (C-3), 148.30 (C-5), 154.14 (C-9), 156.21 (C-4a), 156.48 (C-7), 164.27 (CO); HRMS Calcd. for $\mathrm{C}_{19} \mathrm{H}_{18} \mathrm{~N}_{6} \mathrm{O}_{2}: 362.1491$, found: 362.1478 .

g) Ethyl-5-ethyl-7-imino-3-methyl- $N^{1}$-phenyl-1,7-dihydropyrazolo $\left[3^{\prime}, 4^{\prime}: 4,5\right]$ pyrimido[1,6-a]pyrimidine-6carboxylate $5 g$ Yield $69 \%$; mp $181{ }^{\circ} \mathrm{C}$; IR $\left(\mathrm{cm}^{-1}\right)$; $v_{\mathrm{NH}} 3081 ; v_{\mathrm{CO}} 1706 ; v_{\mathrm{C}=\mathrm{N}} 1434,1493,1589 ; \mathrm{RMN}^{1} \mathrm{H}$ $(\delta \mathrm{ppm}, \mathrm{DMSO}) 1.06\left(3 \mathrm{H}, \mathrm{t}, J=7.1 \mathrm{~Hz}, \mathrm{CH}_{3}\right) ; 1.34$ $\left(3 \mathrm{H}, \mathrm{t}, J=7.0 \mathrm{~Hz}, \mathrm{CH}_{3}\right) ; 1.97(2 \mathrm{H}, \mathrm{q}, J=7.1 \mathrm{~Hz}$, $\left.\mathrm{CH}_{2}\right) ; 2.63\left(3 \mathrm{H}, \mathrm{s}, \mathrm{CH}_{3}\right) ; 4.03(2 \mathrm{H}, \mathrm{q}, J=7.0 \mathrm{~Hz}$, $\left.\mathrm{CH}_{2}\right) ; 7.49\left(1 \mathrm{H}, \mathrm{t}, J=7.3 \mathrm{~Hz}, \mathrm{ArH}_{4}\right) ; 7.63(2 \mathrm{H}, \mathrm{t}$, $J=7.3 \mathrm{~Hz}, \mathrm{ArH}_{3}$ and $\left.\mathrm{ArH}_{5}\right) ; 8.03(2 \mathrm{H}, \mathrm{d}, J=7.3 \mathrm{~Hz}$, $\mathrm{ArH}_{2}$ and $\left.\mathrm{ArH}_{6}\right) ; 9.57\left(1 \mathrm{H}, \mathrm{s}, \mathrm{H}_{9}\right) ; 11.96(1 \mathrm{H}, \mathrm{s}, \mathrm{NH})$. $\mathrm{RMN}^{13} \mathrm{C}(\delta \mathrm{ppm}, \mathrm{DMSO}) 11.26\left(\mathrm{CH}_{3}\right) ; 14.03\left(\mathrm{CH}_{3}\right)$; $14.07\left(\mathrm{CH}_{3}\right) ; 30.19\left(\mathrm{CH}_{2}\right) ; 67.92\left(\mathrm{CH}_{2}\right) ; 105.58$ (C-6); 114.96 (C-3a); 120.64 (C-2' and C-6'), 125.99 $\left(\mathrm{C}-4^{\prime}\right), 129.69\left(\mathrm{C}-3^{\prime}\right.$ and $\left.\mathrm{C}-5^{\prime}\right), 139.45\left(\mathrm{C}-1^{\prime}\right), 143.25$ (C-10a),154.76 (C-3), 156.97 (C-5), 159.15 (C-9), 162.04 (C-4a), 162.50 (C-7), 164.09 (CO); HRMS Calcd. for $\mathrm{C}_{20} \mathrm{H}_{20} \mathrm{~N}_{6} \mathrm{O}_{2}: 376.1648$, found 376.1621.

h) Ethyl-7-imino- $N^{1}$-phenyl-1,7-dihydropyrazolo $\left[3^{\prime}, 4^{\prime}\right.$ : 4,5]pyrimido[1,6-a]pyrimidine carboxylate $5 \boldsymbol{h}$ Yield $89 \%$; mp $184{ }^{\circ} \mathrm{C}$; IR $\left(\mathrm{cm}^{-1}\right) ; v_{\mathrm{NH}} 3227 ; v_{\mathrm{CO}} 1710 ; v_{\mathrm{C}=\mathrm{N}}$ 1539, 1552, 1574.17; RMN ${ }^{1} \mathrm{H}(\delta$ ppm, DMSO) 1.29 $\left(3 \mathrm{H}, \mathrm{t}, J=7.0 \mathrm{~Hz}, \mathrm{CH}_{3}\right) ; 4.24(2 \mathrm{H}, \mathrm{q}, J=7.0 \mathrm{~Hz}$, $\left.\mathrm{CH}_{2}\right) ; 7.37\left(1 \mathrm{H}, \mathrm{t}, J=7.3 \mathrm{~Hz}, \mathrm{ArH}_{4}\right) ; 7.55(2 \mathrm{H}, \mathrm{t}$, $J=7.3 \mathrm{~Hz}, \mathrm{ArH}_{3}$ and $\left.\mathrm{ArH}_{5}\right) ; 8.14(2 \mathrm{H}, \mathrm{d}, J=7.3 \mathrm{~Hz}$, $\mathrm{ArH}_{2}$ and $\left.\mathrm{ArH}_{6}\right) ; 8.75\left(1 \mathrm{H}, \mathrm{s}, \mathrm{H}_{5}\right) ; 8.83\left(1 \mathrm{H}, \mathrm{s}, \mathrm{H}_{9}\right) ; 9.18$ $\left(1 \mathrm{H}, \mathrm{s}, \mathrm{H}_{3}\right) ; 12.11(1 \mathrm{H}, \mathrm{s}, \mathrm{NH}) . \mathrm{RMN}^{13} \mathrm{C}(\delta \mathrm{ppm}, \mathrm{DMSO})$ $14.11\left(\mathrm{CH}_{3}\right) ; 61.36\left(\mathrm{CH}_{2}\right) ; 103.83(\mathrm{C}-6) ; 114.46(\mathrm{C}-$ 3a); $120.62\left(\mathrm{C}-2^{\prime}\right.$ and $\left.\mathrm{C}^{\prime} 6^{\prime}\right), 126.73\left(\mathrm{C}-4^{\prime}\right), 129.20\left(\mathrm{C}-3^{\prime}\right.$ 


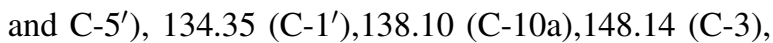
151.37 (C-5), 153.53 (C-9), 154.00 (C-4a), 155.18 (C-7), 163.36 (CO). 120.62-126.73-129.20-134.35, $\mathrm{C}_{17} \mathrm{H}_{14}$ $\mathrm{N}_{6} \mathrm{O}_{2}$, 334.1171; HRMS Calcd. for: $\mathrm{C}_{17} \mathrm{H}_{14} \mathrm{~N}_{6} \mathrm{O}_{2}$ : 334.1178, found: 334.1171.

i) Ethyl-5-methyl-7-imino- $N^{1}$-phenyl-1,7-dihydropyrazolo[3',4':4,5]pyrimido[1,6-a] pyrimidine-6-carboxylate $5 i$ Yield $78 \%$; mp $166{ }^{\circ} \mathrm{C}$; IR $\left(\mathrm{cm}^{-1}\right) ; v_{\mathrm{NH}} 3059 ; v_{\mathrm{CO}}$ $1718 ; v_{\mathrm{C}=\mathrm{N}} 1579,1591,1612 ; \mathrm{RMN}{ }^{1} \mathrm{H}(\delta \mathrm{ppm}, \mathrm{DMSO})$ $1.34\left(3 \mathrm{H}, \mathrm{t}, J=7.0 \mathrm{~Hz}, \mathrm{CH}_{3}\right) ; 1.92(3 \mathrm{H}, \mathrm{s}, J=7.1 \mathrm{~Hz}$, $\left.\mathrm{CH}_{3}\right) ; 4.02\left(2 \mathrm{H}, \mathrm{q}, J=7.0 \mathrm{~Hz}, \mathrm{CH}_{2}\right) ; 7.30(1 \mathrm{H}, \mathrm{t}$, $\left.J=7.3 \mathrm{~Hz}, \mathrm{ArH}_{4}\right) ; 7.61\left(2 \mathrm{H}, \mathrm{t}, J=7.3 \mathrm{~Hz}, \mathrm{ArH}_{3}\right.$ and $\left.\mathrm{ArH}_{5}\right) ; 8.10\left(2 \mathrm{H}, \mathrm{d}, J=7.3 \mathrm{~Hz}, \mathrm{ArH}_{2}\right.$ and $\left.\mathrm{ArH}_{6}\right) ; 9.29$ $\left(1 \mathrm{H}, \mathrm{s}, \mathrm{H}_{3}\right) ; 9.49\left(1 \mathrm{H}, \mathrm{s}, \mathrm{H}_{9}\right) ; 11.95(1 \mathrm{H}, \mathrm{s}, \mathrm{NH}) . \mathrm{RMN}^{13} \mathrm{C}$ ( $\delta$ ppm, DMSO); $15.06\left(\mathrm{CH}_{3}\right) ; 23.14\left(\mathrm{CH}_{3}\right) ; 69.54$ $\left(\mathrm{CH}_{2}\right) ; 102.85(\mathrm{C}-3 \mathrm{a}) ; 117.05(\mathrm{C}-6) ; 121.637\left(\mathrm{C}-2^{\prime}\right.$ and C-6 $\left.{ }^{\prime}\right), 126.41\left(\mathrm{C}-4^{\prime}\right), 128.65\left(\mathrm{C}-3^{\prime}\right.$ and C-5' $), 139.24(\mathrm{C}-$ $\left.1^{\prime}\right), 143.92$ (C-10a),144.17 (C-3), 159.62 (C-5), 161.45 (C-9), 167.12 (C-4a), 167.83 (C-7), 168.28 (CO); HRMS Calcd. for $\mathrm{C}_{18} \mathrm{H}_{16} \mathrm{~N}_{6} \mathrm{O}_{2}$ : 348,1335, found 348, 1274 .

\section{Pharmacology}

Carrageenan (BDH Chemicals Ltd., Poole, England), cimetidine and acetylsalicylic-lysine were purchased from pharmacie Centrale of Tunisia.

\section{Animals}

Adult Male Wistar rats weighing 150-170 g were obtained from Pasteur Institute (Tunis, Tunisia). They were housed in polypropylene cages and left for 2 days for acclimatization to animal room maintained under controlled conditions: a $12 \mathrm{~h}$ light-dark cycle (at $22 \pm 2{ }^{\circ} \mathrm{C}$ ) on standard pellet diet and water ad libitum. Rats were fasted overnight with free access to water before the experiments. Housing conditions and in vivo experiments were approved, according to the guidelines established by the European Union on Animal Care (Communautés Économiques Européennes Council [86/609]).

\section{Carrageenan-induced rat paw oedema}

The anti-inflammatory activity of compounds $(\mathbf{5 a}, \mathbf{b}, \mathbf{f}$, g) on carrageenan-induced rat paw oedema was determined according to Winter et al. (1962). The animals were divided into three groups of six rats each. The control group received intraperitoneally $2.5 \mathrm{ml} / \mathrm{kg}$ of vehicle solution (Tween 80/absolute ethanol/saline solution $(0.9 \%)$ in the ratio $1: 1: 18)$. The reference group received acetylsalicyliclysine $(300 \mathrm{mg} / \mathrm{kg}$ i.p. $)$, and the test groups received compounds 5a, b, f, g (50 and $100 \mathrm{mg} / \mathrm{kg}$, i.p.). After
$30 \mathrm{~min}, 0.05 \mathrm{ml}$ of $1 \%$ carrageenan suspension was injected into the left hind paw. The paw volume up to the tibiotarsal articulation was measured using a plethysmometer (model 7150, UgoBasile, Italy) at $0 \mathrm{~h}\left(V_{0}\right)$ (before carrageenan injection) and 1,3 and $5 \mathrm{~h}$ later $\left(V_{\mathrm{T}}\right)$ (after carrageenan injection). Paw swelling was determined for each rat and the difference between $V_{\mathrm{T}}$ and $V_{0}$ was taken as the oedma value. The percent inhibition was calculated according to the following formula:

$$
\begin{aligned}
& \% \text { Inhibition: }\left[\left(V_{\mathrm{T}}-V_{0}\right)_{\text {control }}-\left(V_{T}-V_{0}\right)_{\text {treated }}\right] \\
& \times 100 /\left(V_{\mathrm{T}}-V_{0}\right)_{\text {control }}
\end{aligned}
$$

\section{Gastroprotective activity}

The gastroprotective activity of pyrazolopyrimidopyrimidines 5a, b, f, g was studied in $150 \mathrm{mM} \mathrm{HCl} / \mathrm{EtOH}-$ induced gastric ulcer (Hara and Okabe, 1985). Rats were fasted for $24 \mathrm{~h}$ prior receiving any treatment and were divided into six groups of six animals each. Group I was kept as control group and received the vehicle (Tween 80/Absolute ethanol/Saline solution (0.9\%): 1/1/18). Group II and III received compound 5a $(50,100 \mathrm{mg} / \mathrm{kg}$, i.p.), respectively, and Group IV and V received compound 5b $(50,100 \mathrm{mg} / \mathrm{kg}$, i.p.), respectively. Group VI and VII received compound $\mathbf{5 f}(50,100 \mathrm{mg} / \mathrm{kg}$, i.p.), respectively, and group VIII and IX received compound 5g (50, 100 $\mathrm{mg} / \mathrm{kg}$, i.p.), respectively. Group $\mathrm{X}$ received cimetidine (100 mg/kg, i.p.) as reference drug. After $30 \mathrm{~min}$, all groups were orally treated with $1 \mathrm{ml} / 100 \mathrm{~g}$ of $150 \mathrm{mM}$ $\mathrm{HCl} / \mathrm{EtOH}(40: 60$, v/v) solution for gastric ulcer induction. Animals were sacrificed $1 \mathrm{~h}$ after the administration of ulcerogenic agent; their stomachs were excised and opened along the great curvature, washed and stretched on cork plates. The surface was examined for the presence of lesions and the extent of the lesions was measured. The summative length of the lesions along the stomach was recorded $(\mathrm{mm})$ as lesion index.

\section{Statistics}

Results are expressed as the mean \pm SEM of six animals per group. The data were analysed using Student's $t$ test, ${ }^{*} p<0.05, * * p<0.01$ and $* * * p<0.001$ was considered significant.

\section{Results and discussion}

\section{Chemistry}

The synthetic routes to target compounds $5 \mathbf{a}-\mathbf{i}$ are outlined in Scheme 1. The 5-amino-4-cyano- $N^{1}$-phenylpyrazole 2, 
Scheme 1 Synthetic procedure of compounds $\mathbf{5 a}-\mathbf{i}$. Reagents: $i \mathrm{H}_{2} \mathrm{~N}-\mathrm{NHPh}, \mathrm{CH}_{3} \mathrm{CO}_{2} \mathrm{H}$, $\mathrm{CH}_{3} \mathrm{CO}_{2} \mathrm{H} ;$ ii $\mathrm{R}_{2} \mathrm{C}(\mathrm{OEt})_{3}$, $\mathrm{CH}_{3} \mathrm{CO}_{2} \mathrm{H}$; iii $\mathrm{NH}_{3}$; iv<smiles>[Y]/C(C#N)=C(\[R3])OCC</smiles><smiles>[R]C(=Nc1c(C#N)c([R])nn1-c1ccccc1)OCC</smiles>

2

$$
\begin{aligned}
& \mathrm{R}_{1}: \mathrm{H}, \mathrm{Me}, \\
& \mathrm{R}_{2}: \mathrm{H}, \mathrm{Me} \\
& \mathrm{R}_{3}: \mathrm{H}, \mathrm{Me}, \mathrm{Et} \\
& \mathrm{Y}: \mathrm{CN}, \mathrm{CO}_{2} \mathrm{Et}
\end{aligned}
$$<smiles>[R]c1nc2c3c([R])nn(-c4ccccc4)c3nc([R])n2c(=N)c1[Y]</smiles>

5a-i

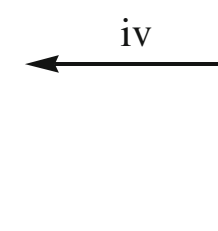<smiles>[R]c1nc(N)c2c([R])nn(-c3ccccc3)c2n1</smiles>

used as a starting material, was prepared in two steps following a similar method reported by Petrie et al. (1985), Anderson et al., (1990), Aggarwal et al., (2011). The first step involves acid-catalysed condensation of orthoester with malonate to form ethoxymethylene malononitrile $\mathbf{1}$. This later reacts then with substituted hydrazine to give the aminocyanopyrazole $\mathbf{2}$. Treatment of $\mathbf{2}$ with orthoester in the presence of catalytic amount of acid furnished the corresponding cyano-pyrazoloimidates $\mathbf{3}$ which subsequently were transformed to the corresponding amino pyrazolopyrimidines 4 (Booth et al., 1999; Gupta et al., 2008; OliveiraCampos et al., 2007; Bakavoli et al., 2010) upon treatment with ammoniac. Reaction of compound $\mathbf{4}$ with ketene ethoxymethylene compounds $\mathbf{1}$ in ethanol in presence of catalytic amount of acid furnished the desired 6-cyano-1,7dihydropyrazolo $\left[3^{\prime}, 4^{\prime}: 4,5\right]$ pyrimido $[1,6-a]$ pyrimidine $\mathbf{5 a - e}$ in $70 \%$ yield as a yellow solid. The same procedure gave a crystalline ethyl-1,7-dihydro pyrazolo $\left[3^{\prime}, 4^{\prime}: 4,5\right]$ pyrimido [1,6-a]pyrimidine-6-carboxylate $\mathbf{5 f - i}$ from ethyl-2-cyano-3- ethoxyalkyl-2-enoate in $80 \%$ yield. Scheme 1 shows the synthetic strategy to obtain the target compounds by the four-steps method, yielding the compounds with structure 5a-i listed in Table 1.

It is interesting to note that time reaction and yield of products are directly related to the nature of substituent $\left(\mathrm{R}_{3}\right.$ and $\left.\mathrm{Y}\right)$. The yields of compounds $\mathbf{5} \boldsymbol{h}$ and $\mathbf{5 d}$ are 89 and $77 \%$, respectively. Hydrogen substituent $\mathrm{R}^{3}$ gave superior yields in short time. In all cases, reaction leads to pyrazolo pyrimido pyrimidine only when $\mathrm{R}^{1}$ or $\mathrm{R}^{2}$ is a hydrogen atom. However, steric effect decreased yields of the reaction, as in the case of $\mathbf{5 g}$, and may even prevent the progress of the reaction when $R^{2}$ and $R^{3}$ are methyl groups. Analysis of the NMR and IR spectra indicated that compounds $\mathbf{5 f}-\mathbf{i}$ has ester functional group in their structures so ethoxymethylene cyanoacetate reacts with pyrazolopyrimidine and in both cases $\mathrm{Y}$ is $\mathrm{CN}$ or $\mathrm{CO}_{2} \mathrm{Et}$, nitrogen attacked on the nitrile function as the first attack.
Table 1 Synthesis of 7-imino$N^{1}$-phenyl-1,7-dihydro pyrazolo $\left[3^{\prime}, 4^{\prime}: 4,5\right]$ pyrimido $[1,6-a]$ pyrimidine $\mathbf{5 a}-\mathbf{i}$

\begin{tabular}{lllllll}
\hline Compounds & $\mathrm{R}_{1}$ & $\mathrm{R}_{2}$ & $\mathrm{R}_{3}$ & $\mathrm{Y}$ & Yields (\%) & Reaction time (h) \\
\hline $\mathbf{5 a}$ & $\mathrm{CH}_{3}$ & $\mathrm{H}$ & $\mathrm{H}$ & $\mathrm{CN}$ & 68 & 24 \\
$\mathbf{5 b}$ & $\mathrm{CH}_{3}$ & $\mathrm{H}$ & $\mathrm{CH}_{3}$ & $\mathrm{CN}$ & 54 & 71 \\
$\mathbf{5 c}$ & $\mathrm{H}$ & $\mathrm{CH}_{3}$ & $\mathrm{H}$ & $\mathrm{CN}$ & 71 & 24 \\
$\mathbf{5 d}$ & $\mathrm{H}$ & $\mathrm{H}$ & $\mathrm{H}$ & $\mathrm{CN}$ & 77 & 5 \\
$\mathbf{5 e}$ & $\mathrm{H}$ & $\mathrm{H}$ & $\mathrm{C}_{2} \mathrm{H}_{5}$ & $\mathrm{CN}$ & 70 & 48 \\
$\mathbf{5 f}$ & $\mathrm{CH}_{3}$ & $\mathrm{H}$ & $\mathrm{CH}_{3}$ & $\mathrm{CO}_{2} \mathrm{Et}$ & 71 & 75 \\
$\mathbf{5 g}$ & $\mathrm{CH}_{3}$ & $\mathrm{H}$ & $\mathrm{C}_{2} \mathrm{H}_{5}$ & $\mathrm{CO}_{2} \mathrm{Et}$ & 69 & 84 \\
$\mathbf{5 h}$ & $\mathrm{H}$ & $\mathrm{H}$ & $\mathrm{H}$ & $\mathrm{CO}_{2} \mathrm{Et}$ & 89 & 7 \\
$\mathbf{5 i}$ & $\mathrm{H}$ & $\mathrm{H}$ & $\mathrm{CH}_{3}$ & $\mathrm{CO}_{2} \mathrm{Et}$ & 78 & 24 \\
\hline
\end{tabular}


Fig. 1 Anti-inflammatory effect of the intraperitoneal administration of $\mathbf{5 a}, \mathbf{b}, \mathbf{f}, \mathbf{g}$ and of the reference drug (acetylsalicylic-lysine: ASL) in carrageenan-induced rat paw oedema. The values represent the means difference of volume of paw $\pm \operatorname{SEM}(n=6)$. $* p<0.01$ and $* * p<0.001$ significantly different from the control group

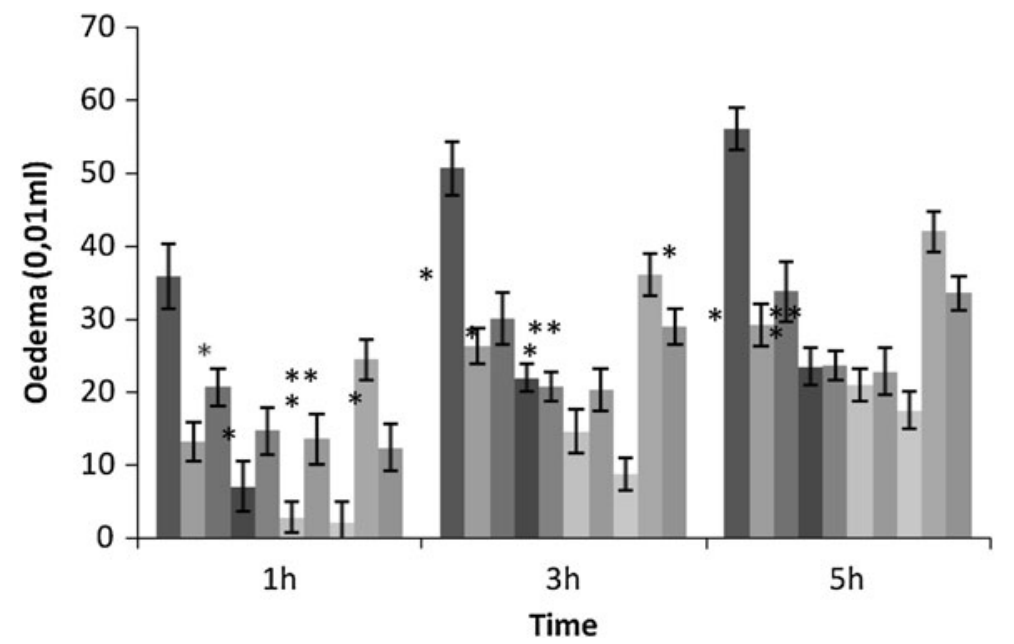

vehicle

ASL

- $5 \mathrm{a}(50 \mathrm{mg} / \mathrm{kg})$

- $5 \mathrm{a}(100 \mathrm{mg} / \mathrm{kg})$

- $5 \mathrm{~b}(50 \mathrm{mg} / \mathrm{kg})$

$5 \mathrm{~b}(100 \mathrm{mg} / \mathrm{kg})$

$5 \mathrm{f}(50 \mathrm{mg} / \mathrm{kg})$

$5 \mathrm{f}(100 \mathrm{mg} / \mathrm{kg})$

$5 \mathrm{~g}(50 \mathrm{mg} / \mathrm{kg})$

$5 \mathrm{~g}(100 \mathrm{mg} / \mathrm{kg})$

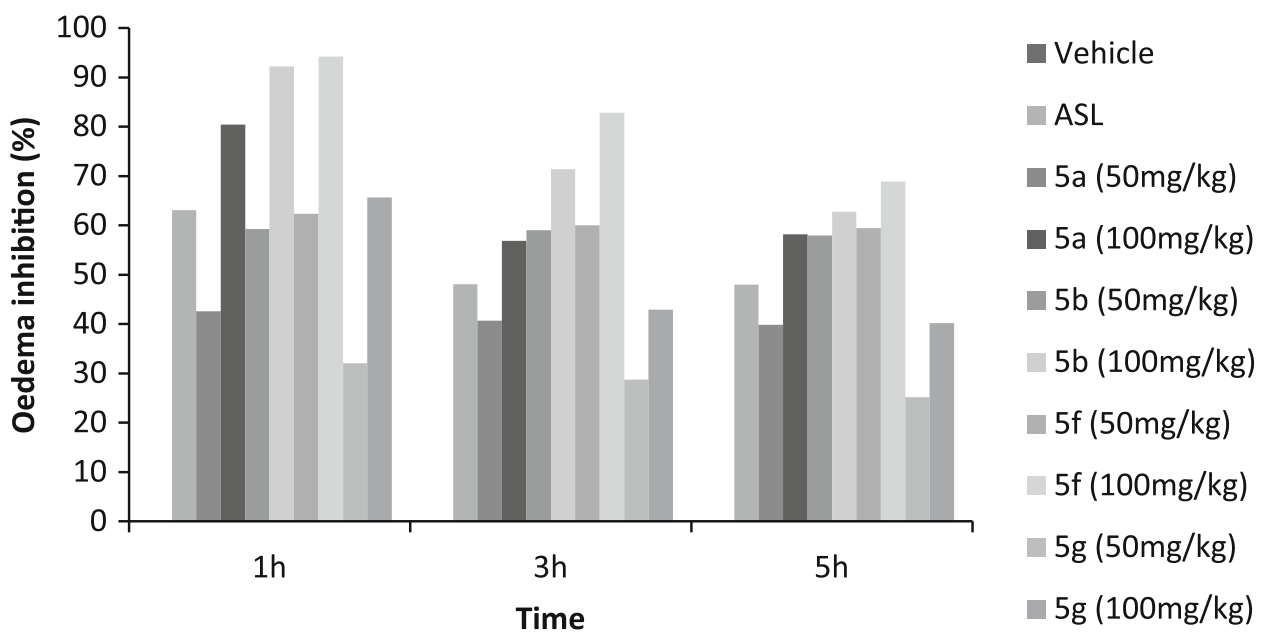

Fig. 2 Percentage inhibition of the oedema after the intraperitoneal administration of $\mathbf{5 a}, \mathbf{b}, \mathbf{f}, \mathbf{g}$ and the reference drug (acetylsalicylic-lysine: ASL) in carrageenan-induced rat paw oedema

\section{Biological activity}

\section{Anti-inflammatory and gastroprotective activities of compounds $\mathbf{5 a}, \boldsymbol{b}, \boldsymbol{f}, \boldsymbol{g}$}

The pyrazolopyrimidine derivatives are a well-known class of NSAIDs with several products in market (Russo et al., 1992; El-Kateb et al., 2012) (Figs. 1, 2).

The structure-activity relationships (SAR) for these compounds have been extensively explored for optimization of anti-inflammatory activity last three decades, since this class was introduced (Lombardino and Wiseman, 1972; Farré et al., 1986; Berq et al., 1999; Lee et al., 1999). In continual efforts to find potentially safer and more efficacious parent agents through further exploration of SAR of this class, we decided to study the pharmacological profiles of compounds $\mathbf{5 a}, \mathbf{b}, \mathbf{f}, \mathbf{g}$ belonging to pyrazolopyrimidopyrimidine family. We examined the effect of modification of the electronic nature of substituents on various portions of type NSAIDs. For this objective the hydrogen atom (position 5) is replaced by methyl or ethyl group, even and for more important anti-inflammatory activity, the cyano function is replaced by ester function.

Table 2 reveals the results of the intraperitoneal administration of the compounds $\mathbf{5 a}, \mathbf{b}, \mathbf{f}, \mathbf{g}$ in carrageenaninduced rat paw oedema. The compounds $\mathbf{5 a}, \mathbf{b}, \mathbf{f}, \mathbf{g}$ tested at 50 and $100 \mathrm{mg} / \mathrm{kg}$, i.p. produced a significant reduction of the oedema throughout the entire period of observation in a dose-dependent manner. The highest reduction of the oedema was at $3 \mathrm{~h}$ after carrageen injection with a percent inhibition ranged, from 40.64 to $56.81 \%$ for compound 5a, from 58.98 to $71.36 \%$ for compound $\mathbf{5 b}$, from 60.02 to $82.83 \%$ for compound $\mathbf{5 f}$ and from 28.75 to $42.87 \%$ for compound $\mathbf{5 g}$, whereas the reference drug (acetylsalicyliclysine, $300 \mathrm{mg} / \mathrm{kg}$, i.p.) produced $48.03 \%$ reduction in paw volume. The influence of the substituent $R_{2}$ on activity is remarkable. Compound $\mathbf{5 a}$ is less potent than the 5-methyl derivatives $\mathbf{5 b}$, so a methyl group linked to the pyrimidine cycle increases the activity compared to the case of a hydrogen atom. At the same dose $(100 \mathrm{mg} / \mathrm{kg})$, compound $\mathbf{5 b}$ produced $71.36 \%$ inhibition of oedema against $56.81 \%$ for $\mathbf{5 a}$. In addition, the compound $\mathbf{5 f}$ is 
Table 2 Anti-inflammatory effect of the intraperitoneal administration of $\mathbf{5 a}, \mathbf{b}, \mathbf{f}, \mathbf{g}$ and of the reference drug (acetylsalicylic-lysine: ASL) in carrageenan-induced rat paw oedema

\begin{tabular}{|c|c|c|c|c|c|c|c|}
\hline \multirow[t]{2}{*}{ Sample } & \multirow{2}{*}{$\begin{array}{l}\text { Dose } \\
(\mathrm{mg} / \mathrm{kg})\end{array}$} & \multicolumn{3}{|c|}{ Oedema $\left(10^{-2} \mathrm{ml}\right)($ mean \pm SEM $)$} & \multicolumn{3}{|c|}{ Oedema inhibition $(\%)$} \\
\hline & & $1 \mathrm{~h}$ & $3 \mathrm{~h}$ & $5 \mathrm{~h}$ & $1 \mathrm{~h}$ & $3 \mathrm{~h}$ & $5 \mathrm{~h}$ \\
\hline Vehicle $(2,5 \mathrm{ml} / \mathrm{kg})$ & - & $35.87 \pm 4.48$ & $50.66 \pm 3.68$ & $56.04 \pm 2.91$ & - & - & - \\
\hline $\begin{array}{l}\text { Acetylsalicylic-lysine } \\
\text { (reference drug) }\end{array}$ & 300 & $13.23 \pm 2.69 * *$ & $26.32 \pm 2.44^{* *}$ & $29.15 \pm 2.87 * *$ & 63.10 & 48.03 & 47.98 \\
\hline \multirow[t]{2}{*}{$5 \mathbf{a}$} & 50 & $20.59 \pm 2.51^{*}$ & $30.07 \pm 3.51^{*}$ & $33.73 \pm 4.16^{*}$ & 42.59 & 40.64 & 39.8 \\
\hline & 100 & $7.01 \pm 3.41^{* *}$ & $21.88 \pm 1.89^{* *}$ & $23.45 \pm 2.5^{* *}$ & 80.44 & 56.81 & 58.15 \\
\hline \multirow[t]{2}{*}{$\mathbf{5 b}$} & 50 & $14.62 \pm 3.21^{*}$ & $20.78 \pm 2 *$ & $23.56 \pm 2 *$ & 59.25 & 58.98 & 57.95 \\
\hline & 100 & $2.81 \pm 2.06^{* * *}$ & $14.51 \pm 2.98 * * *$ & $20.86 \pm 2.21 * * *$ & 92.17 & 71.36 & 62.76 \\
\hline \multirow[t]{2}{*}{$\mathbf{5 f}$} & 50 & $13.51 \pm 3.4^{* *}$ & $20.25 \pm 2.8 * *$ & $22.74 \pm 3.2 * *$ & 62.31 & 60.02 & 59.42 \\
\hline & 100 & $2.07 \pm 2.8^{* * *}$ & $8.69 \pm 2.3 * * *$ & $17.45 \pm 2.5^{* * *}$ & 94.22 & 82.83 & 68.85 \\
\hline \multirow[t]{2}{*}{$5 g$} & 50 & $24.37 \pm 2.7 *$ & $36.09 \pm 2.9 *$ & $41.95 \pm 2.8$ & 32.04 & 28.75 & 25.13 \\
\hline & 100 & $12.31 \pm 3.2^{* *}$ & $28.94 \pm 2.4^{*}$ & $33.52 \pm 2.3$ & 65.66 & 42.87 & 40.18 \\
\hline
\end{tabular}

The values represent the mean difference of volume of paw $\pm \operatorname{SEM}(n=6)$

$* p<0.05, * * p<0.01, * * * p<0.001$ significantly different from control group

more potent than the ethyl derivatives 5g, so an ethyl group linked to the pyrimidine cycle decreases the activity compared to the methyl group.

On the other hand, mucosal erosion and ulceration are produced by most NSAIDs with varying degrees. Inhibition of synthesis of gastroprotective prostaglandins $\left(\mathrm{PGE}_{2}\right)$ is clearly involved (Nezamis et al., 1967) and due to the inhibition of the constitutive isoform COX-1 (Main and Whittle, 1973; Cryer and Feldman, 1992). Thus, deficiency of PGs reduces the mucosal secretions along with hydrogen carbonate that ultimately aggravates the lethal effects of acid on the stomach lining leading to mucosal damage (Fig. 3).

The results of gastroprotective activity of compounds $\mathbf{5 a}$, $\mathbf{b}, \mathbf{f}, \mathbf{g}$ on gastric ulcer induced by $\mathrm{HCI} /$ ethanol solution are shown in Table 3. Oral administration of the ulcerogenic agent to the control group clearly showed a mucosal damage characterized by multiple haemorrhage red bands of different sizes along the long axis of the glandular stomach as described in other studies (Shay et al., 1945; Yassir et al., 1999). When we compared the gastroprotective activity of compounds $\mathbf{5 a}, \mathbf{b}, \mathbf{f}, \mathbf{g}$ we observed that

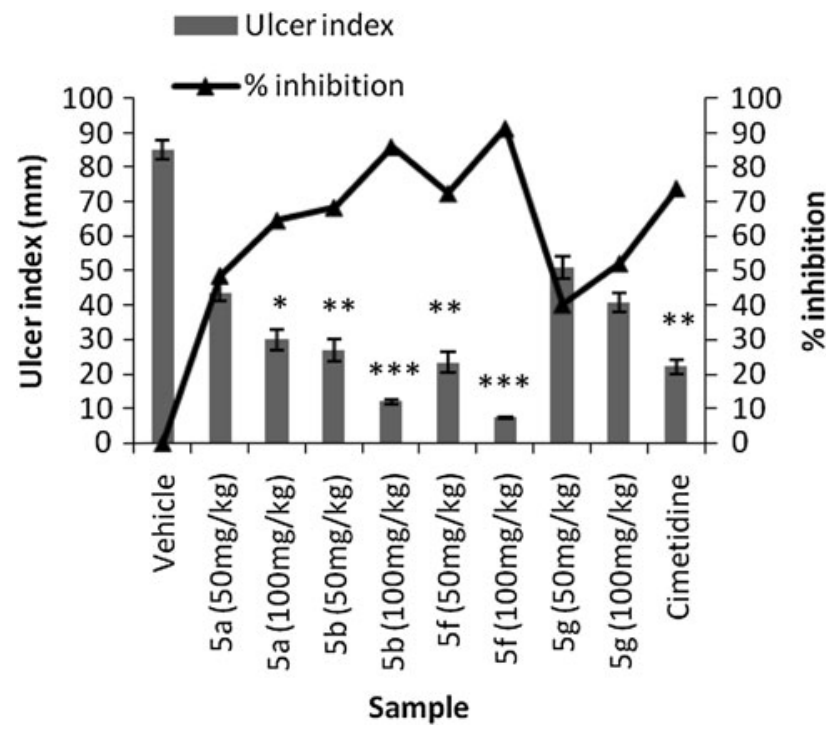

Fig. 3 Effect of compounds $\mathbf{5 a}, \mathbf{b}, \mathbf{f}, \mathbf{g}$ and the reference drug (cimetidine) on gastric ulcer induced by $\mathrm{HCl} /$ ethanol in rats. Data expressed as mean \pm SEM $(n=6) . * p<0.05, \quad * * p<0.01$, $* * * p<0.001$ significantly different from control group
Table 3 Effect of compounds 5a, b, f, $\mathbf{g}$ and the reference drug (cimetidine) on gastric ulcer induced by $\mathrm{HCl} / \mathrm{ethanol}$ in rats

\begin{tabular}{lccl}
\hline Treatment & $\begin{array}{l}\text { Dose } \\
(\mathrm{mg} / \mathrm{kg})\end{array}$ & $\begin{array}{l}\text { Ulcer index } \\
(\mathrm{mm})\end{array}$ & $\begin{array}{l}\text { Inhibition } \\
(\%)\end{array}$ \\
\hline $\begin{array}{l}\text { Vehicle }(2.5 \mathrm{ml} / \mathrm{kg}) \\
(\text { control })\end{array}$ & - & $85 \pm 2.82$ & - \\
Compounds & & & \\
$\mathbf{5 a}$ & 50 & $43.66 \pm 2.58$ & 48.63 \\
& 100 & $30 \pm 3.03^{*}$ & 64.7 \\
$\mathbf{5 b}$ & 50 & $26.83 \pm 3.43^{* *}$ & 68.43 \\
& 100 & $11.83 \pm 0.75^{* * *}$ & 86.08 \\
$\mathbf{5 f}$ & 50 & $23.34 \pm 2.9^{* *}$ & 72.53 \\
& 100 & $7.29 \pm 0.3^{* * *}$ & 91.42 \\
$\mathbf{5 g}$ & 50 & $50.81 \pm 3.2$ & 40.22 \\
& 100 & $40.65 \pm 2.8$ & 52.17 \\
Cimétidine (reference & 100 & $22.07 \pm 2.12^{* *}$ & 74.03 \\
drug) & & & \\
\end{tabular}

Data expressed as mean $\pm \operatorname{SEM}(n=6)$

$* p<0.05, * * p<0.01, * * * p<0.001$ significantly different from control group 
pyrazolopyrimidopyrimidine $\mathbf{5 b}(100 \mathrm{mg} / \mathrm{kg})$ demonstrated the higher significant inhibition of gastric lesion (91, $42 \%)$.

In conclusion, we have synthesized a new series of 1,7dihydropyrazolo $\left[3^{\prime}, 4^{\prime}: 4,5\right]$ pyrimido $[1,6-a]$ pyrimidine 5a-i derivatives. The yield of the reaction seems to be significantly influenced by the nature of substituent. The highest yield is obtained for more hydrogen atom substituent. However, test (or experimental) compounds 5a, b, f showed that the methyl group increases the anti-inflammatory activity, contrary to ethyl group which decreases this activity. The same interpretation is found with gastroprotective effect. Indeed, our results on the gastroprotective effects of compounds 5a, b, $\mathbf{f}$ compared with cimetidine indicate that replacement of hydrogen by methyl reduces the gastrointestinal adverse effects.

Open Access This article is distributed under the terms of the Creative Commons Attribution License which permits any use, distribution, and reproduction in any medium, provided the original author(s) and the source are credited.

\section{References}

Aggarwal R, Kumar V, Kumar R, Singh SP (2011) Approaches towards the synthesis of 5-aminopyrazoles. Beilstein $\mathrm{J}$ Org Chem 7:179-197. doi:10.3762/bjoc.7.25

Allouche F, Chabchoub F, Carta F, Supuran CT (2013) Synthesis of aminocyanopyrazoles via a multi-component reaction and anticarbonic anhydrase inhibitory activity of their sulfamide derivatives against cytosolic and transmembrane isoforms. J Enzyme Inhib Med Chem 28:343-349. doi:10.3109/14756366.2012.720573

Anderson JD, Cottam HB, Larson SB, Nord LD, Revankar GR, Robins RK (1990) Synthesis of certain pyrazolo[3, 4-d]pyrimidin-3-one nucleosides. J Heterocycl Chem 27:439-453. doi:10. 1002/jhet.5570270262

Bakavoli M, Bagherzadeh G, Vaseghifar M, Shiria A, Pordel M, Mashreghi M, Pordeli P, Araghi M (2010) Molecular iodine promoted synthesis of new pyrazolo[3, 4- $d]$ pyrimidine derivatives as potential antibacterial agents. Euro J Med Chem 45:647-650. doi:10.1016/j.ejmech.2009.10.051

Berq J, Fellier H, Christoph T, Grarup J, Stimmeder D (1999) The analgesic NSAID lornoxicam inhibits cyclooxygenase (COX)$1 /-2$, inducible nitric oxide synthase (iNOS), and the formation of interleukin (IL)-6 in vitro. Inflamm Res 48:369-379

Booth BL, Costa FAT, Mahmood Z, Pritchard RG, Proença MF (1999) Synthesis of (Z)-N-(2-amino-1,2-dicyanovinyl)formamide O-alkyloximes and a study of their cyclisation in the presence of base. J Chem Soc Perkin Trans 1:1853-1858

Cryer B, Feldman M (1992) Effects of nonsteroidal anti-inflammatory drugs on endogenous gastrointestinal prostaglandins and therapeutic strategies for prevention and treatment of nonsteroidal anti-inflammatory drug-induced damage. Arch Intern Med 152:1145-1155. doi:10.1001/archinte.1992.00400180017003

El-Kateb AA, Abd El-Rahman NM, Saleh TS, Zeid IF, Mady MF (2012) Microwave-assisted synthesis of novel pyrazole, pyrimidine and pyrazolo[1,5- $a$ ]pyrimidines containing aryl sulfone moiety. Life Sci J 9:711-718

Farré AJ, Colombo M, Fort M, Gutiérrez B, Rodriguez L, Roser R (1986) Pharmacological properties of droxicam, a new nonsteroidal anti-inflammatory agent. Methods Find Exp Clin Pharmacol 8:407-422
Gupta S, Rodrigues LM, Esteves AP, Oliveira-Campos AMF, José Nascimento MS, Nazareth N, Cidade H, Neves MP, Fernandes E, Pinto M, Cerqueira NMFSA, Natercia B (2008) Synthesis of $\mathrm{N}$-aryl-5-amino-4-cyanopyrazole derivatives as potent xanthine oxidase inhibitors. Eur J Med Chem 43:771-780. doi:10.1016/j. ejmech.2007.06.002

Hara N, Okabe S (1985) Effects of gefernate on acute lesions in rats. Folia Pharmacologica Japonica 85:443-448

Lee EB, Known SK, Kim SG (1999) Synthesis and analgesic and antiinflammatory activities of 1,2-benzothiazine derivatives. Arch Pharm Res 22:44-47. doi:10.1007/BF02976434

Lipscomb GR, Wallis N, Armstrong G, Rees WD (1998) Gastrointestinal tolerability of meloxicam and piroxicam: a double blind placebo-controlled study. Br J Clin Pharmacol 46:133-137. doi:10.1046/j.1365-2125.1998.00761.x

Lombardino JG, Wiseman EH (1972) Sudoxicam and related $\mathrm{N}$-heterocyclic carboxamides of 4-hydroxy- $2 \mathrm{H}$-1,2-benzothiazine 1,1-dioxide. Potent nonsteroidal antiinflammatory agents. J Med Chem 15:848-849. doi:10.1021/jm00278a016

Main IHM, Whittle BJR (1973) The effects E and A prostaglandins on gastric mucosal blood flow and acid secretion in the rat. Br J Pharmacol 49:428-436

Moote C (1992) Efficacy of nonsteroidal anti-inflammatory drugs in the management of postoperative pain. Drugs 44:14-30. doi:10. 2165/00003495-199200445-00004

Nezamis JE, Philips JP, Am J (1967) Inhibition of gastric secretion by prostaglandins. Am J Dig Dis 12:1073-1076

Oliveira-Campos AMF, Salaheldin AM, Rodrigues LM (2007) Synthesis of some novel pyrazolo $[3,4-d]$ pyrimidine derivatives. Arkivoc 16:92-100. doi:10.3998/ark.5550190.0008.g10

Petrie CR, Cottam HB, Mekernam PA, Robins RK, Revankar GR (1985) Synthesis and biological activity of 6-azacadeguomycin and certain 3,4,6-trisubstituted pyrazolo[3, 4- $d$ ] pyrimidine ribonucleosides. J Med Chem 28:1010-1016. doi:10.1021/ jm00146a007

Russo F, Guccione S, Romeo G, Monsu L, Pucci S, Caruso A, Cutuli V, Roxas MA (1992) Synthesis and pharmacological properties of pyrazolotriazolopyrimidine derivatives. Eur J Med Chem 27:73-80

Shay JP, Komaov SA, Fels SS, Meranze D, Grunstein M, Simpler H (1945) A simple method for the uniform production of gastric ulceration in the rat. Gastroenterology 5:43-61

Simon LS, Lanza FL, Lipsky PE, Hubbard RC, Talwalker S, Schwartz BD, Isakson PC, Geis GS (1998) Preliminary study of the safety and efficacy of SC-58635, a novel cyclooxygenase 2 inhibitor: efficacy and safety in two placebo-controlled trials in osteoarthritis and rheumatoid arthritis, and studies of gastrointestinal and platelet effects. Arthritis Rheum 41:1591-1601

Vane JR, Botting RM (1995) New insights into the mode of action of anti-inflammatory drugs. Inflamm Res 44:1-10. doi:10.1007/ BF01630479

Vane JR, Botting RM (1996) Mechanism of action of antiinflammatory drugs. Scand J Rheumatol 25:9-25

Vane JR, Botting RM, Bakhle YS (1998) Cyclooxygenases 1 and 2. Annu Rev Pharmacol Toxicol 38:97-120. doi:10.1146/annurev. pharmtox.38.1.97

Weitzel KW, Wickman JM, Augustin SG, Strom JG (2000) Zaleplon: a pyrazolo pyrimidine sedative-hypnotic agent for the treatment of insomnia. Clin Ther 22:1254-1267

Winter CA, Risley GA, Nuss GW (1962) Carrageenin-induced edema in hind paw of the rat as an assay for antiiflammatory drugs. Proc Soc Exp Biol Med 111:544-547

Yassir M, Hummadi AM, Najim RA, Farjou IB (1999) A new in vitro model for ethanol induced gastric mucosal damage. Jap J Pharmacol toxicol methods 41:167-172 